\title{
Arte en la ciudad: lenguajes tecnológicos y privacidad ciudadana ${ }^{*}$
}

\author{
DAVID TRUJILLO \\ Artista y profesor de "Instalaciones" y "Arte en la red y aplicaciones multimedia" \\ Facultad de Bellas Artes de Altea, Universidad Miguel Hernández
}

\begin{abstract}
Valorando los diferentes puntos de vista que planteaban el resto de ponencias presentadas a las Jornadas Arte y Ciudad, entendí que la perspectiva más adecuada para completar las Jornadas sería afrontar mi intervención planteando el funcionamiento de la ciudad y sus posibilidades para el artista contemporáneo que utiliza los medios audiovisuales para expresarse.
\end{abstract}

Empecé a dar forma a lo que quería contar teniendo como premisa al público a quien va dirigido y el lugar donde se va a celebrar, la Sala Naranja de la Facultad de Ciencias de la Información, y tenía tres ideas muy claras de lo que quería que fuera mi intervención, la primera, reflexionar con el concepto de la ciudad como "site", segundo el funcionamiento y/o posibilidades de la ciudad para el artista contemporáneo, y tercero, la demostración de lo anterior en un contexto de vídeovigilancia, llamémoslo así, pero sobre todo lo que

\footnotetext{
* Texto revisado y actualizado de la ponencia presentada en las I Jornadas Arte y Ciudad, Facultad de Ciencias de la Información, UCM, Madrid 25 y 26 de mayo de 2007.
} 
nos interesa es que es un medio o una corriente que utiliza los componentes videográficos, tanto de entrada (cámaras: web, hd, móvil, etc...) y salida (pantallas: lcd, leds, los edificios, el vapor de agua, etc...) de una manera clara, concisa y ejemplificadora para nuestra tesis. También funcionaría el arte sonoro, del que tomamos muchas referencias.

Para entender a la ciudad como "site" deberíamos dar un repaso por la evolución de la escultura en el siglo pasado, citando algunas referencias fundamentales: como la teoría del campo expandido de Rossalind Krauss, la relectura de la misma de José Luis Brea, la pérdida del pedestal e interferencias en el espacio escultórico de Javier Maderuelo, por citar algunas. Y si se quiere por su dimensión temporal (indivisible en el contexto de videovigilancia) desde una perspectiva sonora, con José Iges, o Brandon Labelle, quien en el 2004 publicó un catálogo titulado Site specific sound donde recogía una serie de intervenciones sonoras desde 1998 hasta 2002 englobadas en el Music Sound Festival de Los Angeles. Cada intervención fue creada anualmente como parte del festival sobre la casa y los jardines del Beyond Baroque Literary/Arts Center. Las intervenciones exploran la relaciones sociales y espaciales entre el sonido y la arquitectura del lugar. Intentaré citar sólo los necesarios.

Las intervenciones en los espacios públicos y naturales surgieron como negación del museo. En los años ochenta el museo ya se había desmitificado. El proceso de democratización de la época posmoderna llevó a reconsiderar el papel del usuario y del contexto. La atención se desplazó de las obras de arte autorreferenciales y autónomas para empezar a moverse en una dirección que incluyera al usuario. Las exposiciones se celebraban en lugares cada vez más públicos y menos institucionales, retando así intencionadamente el aura enrarecida del museo tradicional.

Los artistas comenzaron a plantearse tanto la forma de su obra como el contexto en el que las presentaban. Las esculturas autónomas, colocadas arbitrariamente en los espacios públicos, fueron tachadas de obras acontextuales, que parecían tiradas en cualquier sitio. La obra "site-specific" iba a sustituir el uso indiscriminado del arte como complemento pintoresco del tejido urbano. Convencidos de que el arte había sido hasta entonces demasiado exclusivo y 
elitista, los artistas empezaron a trabajar en lugares "públicos" alejados de los límites tradicionalmente más intelectuales y físicos del arte. Los espacios empleados comúnmente por la escena artística podían ser tan variados como hospitales, escuelas, viviendas públicas, iglesias y cárceles.

El paso del museo a la calle, de un espacio institucional a uno de carácter público y no institucional, ha transformado tanto la obra de arte como el papel del artista. La posibilidad de definir un lugar no sólo físicamente sino mediante referencias a su historia, a su significación política o a su importancia para un cierto grupo étnico provoca que los artistas definan nuevos papeles más "públicos" para sí mismos. En teoría, el artista "público" contemporáneo tiende a no concebir y crear sus obras tridimensionales en el entorno protegido de su taller. Trabaja in situ y analiza condiciones básicas del emplazamiento como la escala, el usuario o el potencialmente complejo carácter del contexto. El arte site-specific persigue poner de relieve la percepción consciente del lugar y ratificar los tópicos lazos entre el lugar y su contexto concreto.

Dado que el éxito de la obra depende de la recepción del observador, a quien ahora se dirige la atención del artista, es importante que se defina escrupulosamente el carácter del lugar y de su usuario y que se calcule bien el efecto. Gerhard Bott, en su libro sobre el futuro del museo (1970), ya sostenía que

el arte debe tener una presencia extramuros a fin de disipar el odio de la exclusividad. El arte forma parte de la vida y la vida está expuesta a cambios y a nuevas orientaciones que deben ser visibles y efectivos en todo lugar.

(Schulz-Dornburg, 2002: 8).

Si su obra busca dirigirse a la gente que frecuenta el lugar en cuestión, integrarse de verdad y estar "en contexto", el artista debe convertirse en coordinador, político, diseñador y sociólogo a fin de alcanzar su objetivo. Su papel es ahora multifuncional; su trabajo interdisciplinar, lo mismo que la de su homólogo el arquitecto. Para entender aún más la conceptualización del site en intervención urbana, nos vamos a servir de varias definiciones de Instalación de la artista Concha Jerez, que han sido referenciadas en distintas publi- 
caciones. Partiendo de la definición de Concha Jerez citada por Maderuelo en su Interferencias en el espacio escultórico:

Una INSTALACIÓN es una obra única que se genera a partir de un concepto y/o de una narrativa visual creada por el artista en un espacio concreto. En él se establece una interacción completa entre los elementos introducidos y el espacio considerado como obra total. (Maderuelo, 1990).

Podemos ver que para la existencia de dicho tipo de obras está ligada a su relación especifica con el espacio (más adelante reflexionaremos de la vigencia actual de esta definición ya que actualmente casi todas las instalaciones son itinerantes).

La otra definición de Jerez la encontramos en el texto de José Igés Territorios artísticos para oír y ver:

La instalación surge como una expansión de la dimensionalidad, con la notable diferencia respecto de la escultura de que los ejes respecto a los cuales se organiza la materia no son ya exclusivamente internos a la obra sino también exteriores a ella, pues uno está vinculado al espacio mientras el otro coincide con el meramente constructivo de los elementos que conforman la instalación. (...) una obra es instalación si dialoga con el espacio que la circunda (...) la instalación in situ es la instalación per se, aunque existen instalaciones que se pueden adaptar a distintos espacios. (Iges, 1999).

Ya en esta definición, vemos cómo Concha Jerez deja abierta la posibilidad de que la relación espacial es determinante, pero no exclusiva, ya que este tipo de obras se pueden adaptar a distintos espacios. Podemos decir entonces, que las instalaciones se basan, generalmente, en la creación específica de entornos plurisensoriales, muchas veces objetuales, en los que la propia tridimensionalidad conduce a los artistas a conjugar o a poner el énfasis en aspectos como la dimensión espacial, el recorrido temporal o el carácter procesual e interactivo.

Uno se preguntará por qué utilizo la definición de instalación, pues bien, las instalaciones nacieron englobando la práctica artística realizada para un 
site (un lugar determinado). Más tarde, por motivos diversos como la apropiación de las instituciones y las galerías por dicho arte emergente, se ha desarrollado una nueva fórmula de instalación, aquellas que pueden ser transportables, itinerantes, que además no dialogan con el espacio, aunque sí lo necesiten para su exhibición. Y podemos ir más allá, ya que en la actualidad se realizan obras consideradas (por la gran mayoría) como intervenciones cuando en realidad son instalaciones en espacios urbanos, es decir aquellas piezas que se realizan en un espacio no institucional: por ejemplo un colegio, llamémoslo " $x$ ", pero que se podría realizar en otro colegio " $y$ " de otra ciudad, o zona, sin cambiar ni un ápice su concepto. Aunque necesite de las características arquitectónicas propias de un colegio.

Recapitulando, las intervenciones, serían aquellas que dialogan con: la arquitectura, lo social, lo cultural del lugar, tanto en espacios abiertos (urbanos o naturales) o cerrados, espacios con cualidades o características irrepetibles en otro lugar. Este lugar específico (site) determinará también un contexto, que alterará la lectura de la obra. Las características del site modifican completamente la percepción que podemos tener del hecho artístico.

Volviendo a nuestro planteamiento, la ciudad para un artista funcionaría de tres maneras distintas. Para dicha clasificación me he basado en un fragmento de La ciudad resonante de José Iges:

...más que geometría urbana, en la poética de una hora y un lugar concretos, en el trazado urbanístico o en la red de transportes públicos, encontramos que los artistas han comprendido la ciudad como un incesante emisor de signos, y sus obras han perseguido, mayoritariamente, atrapar esos signos para transgredirlos o trascenderlos, en un contexto así mismo mediático: el de su difusión en espacios públicos de la propia ciudad, en una sala de conciertos o en el medio radio. (Iges, 1997)

La resultante de la relación entre el espacio (urbano o de la naturaleza) y el sonido, ha constituido la base de múltiples propuestas artísticas que ofrecen para nosotros tres perspectivas del uso de la ciudad: dos diferenciadas y otra, que une a las dos. Es decir: el espacio como contenedor de la obra (interven- 
ción, soporte); el espacio como generador de la obra (nos da el material); y el espacio como generador y contenedor de la obra (unión de los dos, a tiempo real o retardado).

La ciudad por tanto funcionaria:

- como continente de la obra: a modo de espacio expositivo, hay de todo, existen artistas que estudian el lugar de intervención, la memoria del lugar, la arquitectura, las necesidades especificas, hay otros que no, ....

- como un generador de signos, datos, información: los artistas recopilan información para realizar sus trabajos que posteriormente presentarán en otros lugares o formatos: instalaciones, videoarte, documentales, cds, etc.

- como generador y continente de la obra: sería una combinación de ambas, la ciudad aporta datos al artista que usa posteriormente volcándolos en la propia ciudad, o por lo menos entorno urbano, en algunos casos manufacturándolos en otros no. En algunos casos, a tiempo real, en otros retardado.

Para mí, la más interesante y la que plantea nuevos campos para investigar conjuntamente con los nuevos materiales escultóricos sería la tercera vía, ya que como veremos introduce el concepto de los espacios blandos ${ }^{1}$, hertziano ${ }^{2}$ de la ciudad.

La elección de la videovigliancia y la privacidad, aunque recurrente, en nuestro caso está justificado; en primer lugar porque se ha convertido en un género con identidad propia (desde el net art y los nuevos medios); en segundo lugar porque se reflexiona teóricamente sobre la ciudad; y el tercero es

\footnotetext{
${ }^{1}$ Un día, durante los meses que pasé en Japón como artista en residencia en la escuela IAMAS, estaba en mi habitación intentando hacer una llamada desde mi móvil, y noté que en algunas partes no tenía prácticamente cobertura, mientras que en otras la señal llegaba con mucha fuerza. Casi podía sentir que el espacio de las ondas era una realidad topográfica diferente, como una capa que se superponía al espacio arquitectónico. Usman Haque. Texto de José Luis de Vicente en Artfutura 2005, Espacio Hertziano, espacios sensibles, blandos. http://www.artfutura.org/02/05devicente.html

${ }^{2}$ Véase también: Anthony Dunne “Hertzian Tales". MIT Press, 2006. Cita actualizada.
} 
porque al ser un tema tecnológico y que va arraigado al proceso de evolución de las tecnologías de comunicación, y nos ofrecen la oportunidad (en el tercer punto) de percibir, de sentir lo invisible de los espacios de flujos ${ }^{3}$ de la ciudad. Además de que existen ejemplos interesantes para explicar las tres posibilidades que nos ofrece la ciudad.

Proliferan las tecnologías de la vigilancia que cruzan los vectores en los que la sociedad contemporánea, sus individuos y sus poderes organizados realizan una mayor participación; estos vectores son: la muerte como crimen, el sexo como pornografía, la identidad como ficción-montaje, el cuerpo como objeto, la memoria como herramienta, la comunicación como progreso, la verdad como discurso, el poder como información y la vigilancia como seguridad. Todo ello expresado en la imagen no sólo como soporte y representación, sino como sustitución de la realidad, imagen como instante puro o presente puro, imagen como prueba judicial, imagen como espectáculo de lo real. Resultado de un poderío y desarrollo económico que necesita de una constante expansión para mantenerse, el tema de la globalización es, además de un asunto eminentemente comercial, la extensión, generalizada de un modo de entender y organizar la vida, en función de unos sistemas de comunicación sin parangón en etapas anteriores de la historia mundial. Las máquinas de visión. Utilizando el poder de la comunicación - de sus códigos tecnológicos, lingüísticos y semánticos - nos sitúan en los simulacros más hiperrealistas de la vigilancia, el control y la seguridad del individuo. En palabras de Verónica Valencia

La vigilancia se expresa en circuitos cerrados de televisión, videoporteros, cajeros automáticos, transmisiones telemáticas, centros comerciales y de entretenimiento, bancos, escuelas, cárceles, instituciones públicas y privadas, calles, plazas, carreteras, tráfico vehicular, seguridad infantil, clima, medio ambiente, hospitales empresas, casas, cualquier espacio que requiera vigilancia ${ }^{4}$.

Por tanto, la ciudad funcionaría como un espacio electrónico de juego y

\footnotetext{
3 Véase también: Manuel Castells / 1997 [orig. en inglés 1996] / La era de la información. Economía, sociedad y cultura. Vol. 1 La sociedad red / Alianza / Capítulo 6: El espacio de los flujos pp:409-462.

4 Valencia, Verónica. "Facilita Sony labor de vigilancia", artículo publicado en ElNorte.com, 10/09/2002.
} 
simulacro de la vigilancia de la intimidad de otros, desde el propio espacio privado del espectador, sin tener que moverse de su casa, así como las apariencias y relatos de la publicidad, determinan cada vez más el espacio privado del individuo como un espacio de simulacros y ficciones de la vida de los otros y, en consecuencia, su creciente pérdida de la intimidad:

Es preciso percibir que la vigilancia y la iluminación se desplazan a su vez de calles y avenidas, en dirección a esa terminal de recepción de anuncios a domicilio que suple a la ciudad, con lo que la esfera privada continúa perdiendo autonomía (Virilio, 1989).

Bentham estableció el principio de que el poder debía ser visible e inverificable. Visibles son las cámaras en los lugares públicos, inverificable es saber si en ese momento se mira a través de ellas y si están grabando. En la actualidad, el efecto de lo panóptico sobre el escenario humano y el mundo garantiza, tal y como sostuvo Michel Foucault con estas palabras "un funcionamiento automático del poder (Foucault, 1982).

En el régimen panóptico, la vigilancia se convierte en autovigilancia, ya que no hay dónde ocultarse, y la "dictadura de la mirada" controla todo espacio público o privado. Una sociedad vigilada es la que ve paradójicamente su espacio público disociado, convertido en escenario de detección, y su anonimato urbano invadido por una mirada permanentemente vigilante, la calle como lugar de observación controlada, como espacio de control.

Para ello veremos un conjunto de obras que se sitúan en los límites disciplinares de la arquitectura, la escultura, la sociología, la informática, entre otras, en el convencimiento de que estas obras son útiles en cuanto que nos desvelan el desarrollo y el devenir del arte en la ciudad. Clasificación de las obras presentadas y comentadas en el interactivo realizado para la ponencia. 
La ciudad: espacio expositivo para las intervenciones audiovisuales.

http://www.madridabierto.com/en/intervenciones-artisticas/2004/elperro.html (Grupo El perro, virtual demolition mobile) http://www.danielcanogar.com/ (Serie clandestinos, 2006). http://www.notbored.org/the-scp.html (The surveillance camera players). http://www.irational.org/heath/cctv sabotage/ (heath bunting). http://openendedgroup.com/index.php/artworks/pedestrian/ (shelley eshkar y paul kaiser).

http://www.sester.net/projects/access/access.html (Marie Sester).

La ciudad: generadora de estímulos, datos, información para la realización de piezas.

http://www.andreazapp.com/ little sister (2000)a cctv online surveillance soap http://www.appliedautonomy.com/isee.html instituto de autonomía aplicada (iaa). isee, 1998-2002.

http://www.evidencelocker.net/story.php Jill Magid. evidence locker, liverpool biennial international 2004 .

http://www.zexe.net $/ \mathrm{madrid} / \mathrm{madrid} /$ intro.php?qt=7.4\&can actual $=$ Antoni Abad. "canal invisible", 2005.

La ciudad: generadora de estímulos, datos, información y a su vez, espacio expositivo para las intervenciones audiovisuales

http://www.lozano-hemmer.com/under scan.php Rafael Lozano-Hemmer. http://www.haque.co.uk/skyear/ Usman Haque. "sky ear", 2003-2004.

http://www.ubermatic.org/life/ Michelle Teran. "life: a user's manual", 20032006. 


\section{BIBLIOGRAFÍA}

FOUCAULT, Michel (1982): Vigilar y castigar. Siglo XXI, Madrid.

IGÉS, José (1997). "La ciudad resonante", Revista Fisuras de la cultura contemporánea. № 5, Las ciudades inasibles, Madrid, pp. 60-78.

IGÉS, José (1999): “Territorios artísticos para oír y ver”, en Catálogo exposición El espacio del sonido - El tiempo de la mirada, Koldo Mitxelena Kulturunea, Diputación Provincial de Gipuzkoa, Donosti/S. Sebastian, pp. 4-28.

MADERUELO, Javier (1990): "Interferencias en el espacio escultórico". Madrid. Espacio de Interferencias. Círculo de Bellas Artes, Madrid.

SCHUlZ-DORnBURG, J. (2002) Arte y arquitectura: nuevas afinidades. Gustavo Gili, Barcelona.

VIRILIO, Paul (1989). La máquina de visión. Cátedra, Madrid. 